Pacific Journal of Mathematics

MITTAG-LEFFLER EXPANSIONS AND SPECTRAL THEORY 


\title{
MITTAG-LEFFLER EXPANSIONS AND SPECTRAL THEORY
}

\author{
ANGUS E. TAYLOR
}

1. Introduction and Summary. Consider a bounded linear operator $A$ acting in a complex Banach space $X$ having some nonzero elements. In this paper we shall assume that $\sigma(A)$, the spectrum of $A$, consists of 0 and the distinct points $\lambda_{1}, \lambda_{2}, \lambda_{3}, \cdots$, where $\lambda_{n} \neq 0$ and $\lambda_{n} \rightarrow 0$ as $n \rightarrow \infty$. We shall denote by $[X]$ the Banach space of all bounded linear operators mapping $X$ into itself, with the usual operator norm. The inverse operator $(\lambda-A)^{-1} \equiv R_{\lambda}(A)$ (the resolvent of $A$ ) is an analytic function from the resolvent set $\rho(A)$ (the complement of $\sigma(A)$ ) to $[X]$. We shall assume that each of the points $\lambda_{n}$ is a simple pole of $R_{\lambda}(A)$. Let $E_{n}$ be the residue of $R_{\lambda}(A)$ at $\lambda_{n}$. Then it is known, from general spectral theory, that

$$
E_{n}^{2}=E_{n}, \quad E_{n} E_{m}=0 \text { if } m \neq n,
$$

and further, that $E_{n} \neq 0, E_{n} \neq I$. It is also important to note that $A E_{n}=E_{n} A$. For these facts and other relevant parts of general theory we refer the reader to Chapter 5 of the author's book [3].

By using the extension of Mittag-Leffler's theorem to vector-valued analytic functions, along with an inversion to convert $R_{\lambda}(A)$ into a meromorphic function, and then converting back again, we find that $R_{\lambda}(A)$ can be expressed in the form

$$
R_{\lambda}(A)=\sum_{n=1}^{\infty} \frac{\lambda_{n}^{v_{n}}}{\lambda^{v_{n}}\left(\lambda-\lambda_{n}\right)} E_{n}+\Phi(\lambda),
$$

where each $U_{n}$ is a nonnegative integer and $\Phi$ is an entire function of $1 / \lambda$. The series involving the $E_{n}$ 's converges (in the uniform operator topology) uniformly on each compact set in the complement of $\sigma(A)$. It turns out that $v_{n}>0$ for all sufficiently large values of $n$. Also, the coefficients in the expansion of $\Phi$ as a power series in $1 / \lambda$ are expressible in terms of $A$ and the $E_{n}$ 's. The details of all this are given in $\S 2$.

The main purpose of this paper is to investigate the particular cases in which $v_{n}$ is the same for all values of $n$. The case $v_{n} \equiv 1$ is the simplest and the most fundamental. In that case the series in (1.2) takes the form

$$
\sum_{n=1}^{\infty} \frac{\lambda_{n}}{\lambda\left(\lambda-\lambda_{n}\right)} E_{n} \equiv \sum_{n=1}^{\infty}\left[\frac{1}{\lambda-\lambda_{n}}-\frac{1}{\lambda}\right] E_{n}
$$

Received July 21, 1959. 
In a certain sense this series supplies a notivation for the entire investigation, for a series of this form occurs in the theory of compact self-adjoint operators in Hilbert space.

Let us suppose, in fact, that $T$ is a compact self-adjoint operator on a complex Hilbert space $X$ of infinite dimension, and let in have distinct nonzero eigenvalues $\lambda_{1} \lambda_{2}, \lambda_{3}, \cdots$. Then, if $E_{n}$ is the projection associated with the spectral set consisting of the single point $\lambda_{n}$, we can represent $T$ in the form

$$
T x=\sum_{n=1}^{\infty} \lambda_{n} E_{n} x .
$$

The resolvent of $T$ has the representation

$$
(\lambda-T)^{-1} x=\frac{1}{\lambda} x+\sum_{n=1}^{\infty}\left[\frac{1}{\lambda-\lambda_{n}}-\frac{1}{\lambda}\right] E_{n} x .
$$

For reference, see $\S 6-4$ in [3]. These series representations are also valid in the forms

$$
\begin{gathered}
T=\sum_{n=1}^{\infty} \lambda_{n} E_{n}, \\
(\lambda-T)^{-1}=\frac{I}{\lambda}+\sum_{n=1}^{\infty}\left[\frac{1}{\lambda-\lambda_{n}}\right] E_{n},
\end{gathered}
$$

with convergence in the sense of the operator norm. To prove (1.5), for example, we make use of the fact that in this situation the $E_{n}$ 's themselves are self-adjoint and mutually orthogonal. Hence

$$
\begin{aligned}
& \left\|T x-\sum_{k=1}^{n} \lambda_{k} E_{k} x\right\|^{2}=\left(\sum_{k=n+1}^{\infty} \lambda_{k} E_{k} x ; \quad \sum_{j=n+1}^{\infty} \lambda_{j} E_{j} x\right) \\
& =\sum_{k=n+1}^{\infty} \lambda_{k}^{2}\left(E_{k} x, x\right)=\sum_{k=n+1}^{\infty} \lambda_{k}^{2}\left\|E_{k} x\right\|^{2} \leqq\|x\|^{2} \sup _{k>n} \lambda_{k}^{2} .
\end{aligned}
$$

The last inequality results from the fact that

$$
\sum_{k=1}^{\infty}\left\|E_{k} x\right\|^{2} \leqq\|x\|^{2}
$$

Since $\lambda_{n} \rightarrow 0$, it follows that

$$
\sup _{|| x|| \leqq 1}\left\|T x-\sum_{k=1}^{n} \lambda_{k} E_{k} x\right\| \rightarrow 0
$$

as $n \rightarrow \infty$; thus (1.5) is proved. The proof of (1.6) is entirely similar in principle.

Let us now return to the general context of (1.2). Our first main theorem is concerned with the case in which $v_{n}=1$ for all values of $n$ 
(actually if we merely assume that $v_{n} \leqq 1$ for all values of $n$, we can alter the function $\Phi$ slightly in (1.2) and thereby arrange to make $v_{n}=1$ for every value of $n$ ).

First MaIn Theorem. Let $A$ be a bounded linear operator on the complex Banach space $X$, with spectrum and spectral projections $\left\{E_{n}\right\}$ as set forth at the beginning of this section. Suppose that the resolvent $R_{\lambda}(A)$ is representable in the form

$$
R_{\lambda}(A)=\sum_{n=1}^{\infty} \frac{\lambda_{n}}{\lambda\left(\lambda-\lambda_{n}\right)} E_{n}+\Phi(\lambda),
$$

where $\Phi$ is an entire function of $1 / \lambda$. Then there exist bounded operators $B_{1}, C_{1}$ such that $B_{1}$ and $C_{1}$ commute with $A, B_{1} C_{1}=C_{1} B_{1}=0, B_{1}$ has the same spectrum as $A, C_{1}$ is quasi-nilpotent and hence has spectrum consisting of the single point 0 , and, finally,

$$
A=B_{1}+C_{1} \text {. }
$$

Furthermore,

$$
\begin{gathered}
B_{1}=\sum_{n=1}^{\infty} \lambda_{n} E_{n}, \\
R_{\lambda}\left(B_{1}\right)=\frac{I}{\lambda}+\sum_{n=1}^{\infty}\left[\frac{1}{\lambda-\lambda_{n}}-\frac{1}{\lambda}\right] E_{n},
\end{gathered}
$$

and

$$
R_{\lambda}(A)=R_{\lambda}\left(B_{1}\right)+R_{\lambda}\left(C_{1}\right)-\frac{I}{\lambda}
$$

The proof is given in $\S 3$.

The second main theorem is concerned with the case in which $v_{n}=p$ (where $p>1$ ) for all values of $n$. In other words, the spectrum of $A$ is as before, but it is assumed that for some $p>1$ the resolvent of $A$ is expressible in the form

$$
R_{\lambda}(A)=\sum_{n=1}^{\infty} \frac{\lambda_{n}^{p}}{\lambda^{p}\left(\lambda-\lambda_{n}\right)} E_{n}+\Phi(\lambda),
$$

where $\Phi$ is an entire function of $1 / \lambda$.

Second Main Theorem. When $R_{\lambda}(A)$ is expressible in the form (1.12), the first main theorem is applicable to the operator $A^{p}$, and the result is that there is a decomposition

$$
A^{p}=B_{p}+C_{p},
$$


where

$$
B_{p}=\sum_{n=1}^{\infty} \lambda_{n}^{n} E_{n}
$$

and the relations between $A^{p}, B_{p}, C_{p}$ are the same as the relations between $A, B_{1}, C_{1}$ in the first main theorem, and

$$
R_{\lambda}\left(B_{p}\right)=\frac{I}{\lambda}+\sum_{n=1}^{\infty}\left[\frac{1}{\lambda-\lambda_{n}^{p}}-\frac{1}{\lambda}\right] E_{n} .
$$

The proof of this second theorem is given in $\S 4$.

Subject to the general conditions on $A$ stated at the outset of the paper, there is a sort of "simple canonical form of order $p$ " which $R_{\lambda}(A)$ may take under certain conditions. It is

$$
R_{\lambda}(A)=\frac{I}{\lambda}+\frac{A}{\lambda^{2}}+\cdots+\frac{A^{p-1}}{\lambda^{p}}+\sum_{n=1}^{\infty} \frac{\lambda_{n}^{p}}{\lambda^{p}\left(\lambda-\lambda_{n}\right)} E_{n} .
$$

The infinite series here may also be written in the form

$$
\sum_{n=1}^{\infty}\left[\frac{1}{\lambda-\lambda_{n}}-\frac{1}{\lambda}-\frac{\lambda_{n}}{\lambda^{2}}-\cdots-\frac{\lambda_{n}^{p-1}}{\lambda^{p}}\right] E_{n} .
$$

When (1.16) holds we also have

$$
A^{p}=\sum_{k=1}^{\infty} \lambda_{k}^{p} E_{k} .
$$

It is shown in $\S 5$ that, conversely, if $v_{n}=p$ for all $n$ (where $p \geqq 1$ ), and if (1.18) holds, then $R_{\lambda}(A)$ can be expressed in the form (1.16).

When (1.16) holds for a certain value of $p$, it also holds for the next larger value of $p$. This is clear from (1.17) and (1.18). If (1.16) holds and if $p$ is minimal-i.e. if (1.16) does not hold with $p$ replaced by a smaller exponent $q$, we shall say that $A$ is of finite type $p$. There is a growth condition on $R_{\lambda}(A)$ which insures that $A$ shall be of finite type not exceeding $p$. This is the subject of our third main theorem.

ThIRd MaIN Theorem. Suppose there exists a sequence $\left\{C_{n}\right\}$ of rectifiable closed Jordan curves of the following sort:

(1) The origin and $\lambda_{n+1}, \lambda_{n+2}, \cdots$ are inside $C_{n}$, but $\lambda_{1}, \cdots, \lambda_{n}$ are outside $C_{n}$;

(2) if $\varepsilon_{n}=\max |t|$ for $t$ on $C_{n}$, then $\varepsilon_{n} \rightarrow 0$ as $n \rightarrow \infty$, and $l\left(C_{n}\right)=$ $O\left(\varepsilon_{n}\right)$, where $l\left(C_{n}\right)$ is the length of $C_{n}$;

(3) if $M_{n}=\max \left\|R_{t}(A)\right\|$ for $t$ on $C_{n}$, then $\varepsilon_{n}^{p+1} M_{n} \rightarrow 0$ as $n \rightarrow \infty$; here $p$ denotes some positive integer.

Under these conditions $A$ is of some finite type not exceeding $p$. 
This theorem is proved in $\S 5$.

The last two sections of the paper $(\S \S 6,7)$ are concerned with operators of the form

$$
B=\sum_{n=1}^{\infty} \lambda_{n} E_{n},
$$

where the series converges in $[X]$, the $E_{n}$ 's are mutually orthogonal non-zero projections, and $\{\lambda\}_{n}$ is a sequence of distinct constants (which must necessarily converge to zero). In $\S 6$ two different kinds of conditions are given which are sufficient to insure that $B$ is of finite type 1. One condition bears on the sequence $\left\{\lambda_{n}\right\}$. It is that the series

$$
\sum_{n=1}^{\infty}\left|\lambda_{n+1}-\lambda_{n}\right|
$$

be convergent. This is of course satisfied if the $\lambda_{n}$ 's approach 0 monotonely along some ray. The other condition bears on the projections $E_{n}$. It is that for all finite sets of constants $c_{1}, \cdots, c_{n}$

$$
\left\|\sum_{k=1}^{n} c_{k} E_{k}\right\| \leqq M \sup _{i}\left|c_{i}\right|
$$

where $M$ is some absolute constant. This condition is satisfied (with $M=1$ ) if in particular the space $X$ is a Hilbert space and the projections are symmetric and mutually orthogonal.

In $\S 7$ some unsettled questions regarding $B$ are raised, for the case in which the foregoing conditions are not satisfied.

Our first main theorem is somewhat reminiscent of Dunford's theory of spectral operators, as developed in [2], because a spectral operator, in Dunford's sense, admits a decomposition as a sum of a spectral operator of scalar type and a quasi-nilpotent operator. However, the operator $B_{1}$ of (1.9) need not be a spectral operator, for examples may be constructed in which the norms $\left\{\left\|E_{n}\right\|\right\}$ form an unbounded sequence.

2. The Mittag-Leffler expansion of the resolvent. Let $f$ be a function which is analytic in the entire complex $z$-plane except for simple poles at $a_{1}, a_{2}, a_{3}, \cdots$, where $a_{n} \neq 0$ and $a_{n} \rightarrow \infty$. The values of $f$ are assumed to be in a complex Banach space. Let the residue of $f$ at $a_{n}$ be $r_{n}$. According to the classical theorem of Mittag-Leffler, whose statement and proof remain valid when the function values are in a Banach space, there exists a sequence $\left\{u_{n}\right\}$ of nonnegative integers such that if

$$
p_{n}(z)=-\frac{r_{n}}{a_{n}}\left[1+\frac{z}{a_{n}}+\cdots+\left(\frac{z}{a_{n}}\right)^{v_{n}}\right] \text {, }
$$

then 


$$
f(z)=\sum_{n=1}^{\infty}\left[\frac{r_{n}}{z-a_{n}}-p_{n}(z)\right]+\phi(z)
$$

where $\phi$ is an entire function of $z$ and the first series in (2.2) converges uniformly on each compact set which contains none of the $a_{n}$ 's.

It may be noted that $p_{n}(z)$ is a partial sum of the Taylor's series of the function

$$
\frac{r_{n}}{z-a_{n}}
$$

when it is expanded in powers of $z$.

Let us now consider $R_{\lambda}(A)$ as a function of $\lambda$, where $A$ is the operator described in the first paragraph of $\S 1$. The function values here are in $[X]$. If we set $z=1 / \lambda$ and $f(z)=R_{\lambda}(A)$, the fact that $R_{\lambda}(A)$ has a simple pole with residue $E_{n}$ at $\lambda_{n}$ is readily found to mean that $f$ has a simple pole of residue $\left(-1 / \lambda_{n}^{2} E_{n}\right)$ at $z=1 / \lambda_{n}$. When we write

$$
z=\frac{1}{\lambda}, \quad a_{n}=\frac{1}{\lambda_{n}}, \quad r_{n}=-\frac{1}{\lambda_{n}^{2}} E_{n}, \quad \Phi(\lambda)=\phi(z)
$$

in (2.2), we find, after some simplification,

$$
R_{\lambda}(A)=\sum_{n=1}^{\infty}\left[\frac{1}{\lambda-\lambda_{n}}-P_{n}(\lambda)\right] E_{n}+\Phi(\lambda),
$$

where $\Phi$ is an entire function of $1 / \lambda$, and

$$
P_{n}(\lambda)=\left\{\begin{array}{l}
\frac{1}{\lambda_{n}}\left[\frac{\lambda_{n}}{\lambda}+\cdots+\left(\frac{\lambda_{n}}{\lambda}\right)^{v_{n}}\right] \text { if } v_{n} \geqq 1, \\
0 \text { if } v_{n}=0 .
\end{array}\right.
$$

It is an easy matter to verify that

$$
\frac{1}{\lambda-\lambda_{n}}-P_{n}(\lambda)=\frac{\lambda_{n}^{v} n}{\lambda^{v} n\left(\lambda-\lambda_{n}\right)}
$$

consequently (2.4) and (1.2) are equivalent.

We shall refer to (2.3) or (1.2) as a Mittag-Leffler development of $R_{\lambda}(A)$. It is not claimed to be unique, since there is considerable freedom in the choice of the integers $v_{1}, v_{2}, \cdots$.

The fact that $R_{\lambda}(A)$ is a resolvent has many implications for the structure of the series (2.3). We shall proceed to explore these implications. 
Lemma 2.1. It follows from (1.2) that $v_{n}>0$ for all sufficiently large values of $n$.

Proof. We have $\left\|E_{n}\right\| \geqq 1$ as a result of the fact that $E_{n}$ is a projection. Now

$$
\lim _{n \rightarrow \infty}\left(\frac{\lambda_{n}}{\lambda}\right)^{v_{n}} \frac{E_{n}}{\lambda-\lambda_{n}}=0
$$

as a result of the convergence of the series in (1.2). Since $\lambda_{n} \rightarrow 0$, the assertion of the lemma must be true, if we are to avoid a contradiction.

Let us now express $\Phi(\lambda)$ in the form

$$
\Phi(\lambda)=\sum_{n=0}^{\infty} \frac{Q_{n}}{\lambda^{n}},
$$

where the series converges for all nonzero values of $\lambda$. We shall see how to obtain information about the $Q_{n}$ 's by contour integration.

Lemma 2.2. The coefficients $Q_{n}$ in (2.5) are given by the formulas

$$
\begin{aligned}
Q_{0} & =0, \\
Q_{1} & =I-\sum_{v_{n}=0} E_{n}, \\
Q_{k+1} & =A^{k}-\sum_{v_{n} \leqq k} \lambda_{n}^{k} E_{n},
\end{aligned}
$$

It is part of the implication here that, if there are infinitely many $v_{n}$ not exceeding $k$, the series in (2.8) is convergent.

Proof. We know that

$$
R_{\lambda}(A)=\frac{I}{\lambda}+\frac{A}{\lambda^{2}}+\frac{A^{2}}{\lambda^{3}}+\cdots
$$

when $|\lambda|>\|A\|$. Hence, integration around a very large circle centered at $\lambda=0$ gives

$$
\frac{1}{2 \pi i} \oint \lambda^{k} R_{\lambda}(A) d \lambda=\left\{\begin{array}{l}
0 \text { if } k=-1, \\
I \text { if } k=0, \\
A^{k} \text { if } k \geqq 1 .
\end{array}\right.
$$

On the other hand, we can compute the integral in (2.10) by using (2.3) and (2.5). The calculations are simple, and formulas (2.6), (2.7) and (2.8) are the results, 
3. The case $v_{n} \leqq 1$ for all $n$. In this section we assume that $v_{n}$ never exceeds 1 . We may just as well assume that $v_{n}=1$ for all values of $n$, for, since there can be only finitely many integers $n$ for which $v_{n}=0$, it is easy to see with the aid of Lemma 2.2 that we can write (3.1) $R_{\lambda}(A)=\frac{I}{\lambda}+\sum_{n=1}^{\infty}\left[\frac{1}{\lambda-\lambda_{n}}-\frac{1}{\lambda}\right] E_{n}+\sum_{n=1}^{\infty} \frac{1}{\lambda^{n+1}}\left[A^{n}-\sum_{k=1}^{\infty} \lambda_{k}^{n} E_{k}\right]$.

Let us define an operator $B_{1}$ by the formula

$$
B_{1}=\sum_{n=1}^{\infty} \lambda_{n} E_{n}
$$

The convergence of this series (in the uniform operator topology) is assured by Lemma 2.2.

Lemma 3.1. $B_{1}$ has the same spectrum as $A$, and

$$
R_{\lambda}\left(B_{1}\right)=\frac{I}{\lambda}+\sum_{n=1}^{\infty}\left[\frac{1}{\lambda-\lambda_{n}}-\frac{1}{\lambda}\right] E_{n} .
$$

Proof. Let us denote by $S_{\lambda}$ the right member of the formula (3.3); it is defined when $\lambda \in \rho(A)$, where $\rho(A)$ denotes the resolvent set of $A$. From (1.1) we see that

$$
E_{m} S_{\lambda}=\frac{E_{m}}{\lambda}+\left(\frac{1}{\lambda-\lambda_{m}}-\frac{1}{\lambda}\right) E_{m}=\frac{E_{m}}{\lambda-\lambda_{m}} .
$$

Moreover, $S_{\lambda}$ commutes with each $E_{n}$ and hence with $B_{1}$. Using (3.4) we see that

$$
B_{1} S_{\lambda}=\sum_{m=1}^{\infty} \lambda_{m} E_{m} S_{\lambda}=\sum_{m=1}^{\infty} \frac{\lambda_{m} E_{m}}{\lambda-\lambda_{m}}
$$

But

$$
S_{\lambda}=\frac{I}{\lambda}+\sum_{n=1}^{\infty} \frac{\lambda_{n} E_{n}}{\lambda\left(\lambda-\lambda_{n}\right)}
$$

Hence

$$
B_{1} S_{\lambda}=\lambda\left(S_{\lambda}-\frac{I}{\lambda}\right)=\lambda S_{\lambda}-I
$$

or

$$
\left(\lambda-B_{1}\right) S_{\lambda}=I
$$

Since $B_{1}$ and $S_{\lambda}$ commute, it now follows that $(\lambda-B)^{-1}$ exists and is 
equal to $S_{\lambda}$ when $\lambda \in \rho(A)$. Hence $\rho(A) \subset \rho\left(B_{1}\right)$.

It remains to prove that $\rho\left(B_{1}\right) \subset \rho(A)$, or what is the same, that $\sigma(A) \subset \sigma\left(B_{1}\right)$. Now, elements $x$ in the range of $E_{n}$ are characterized by the relation $E_{n} x=x$. Since $E_{n} \neq 0$, we can choose an $x$ of this kind such that $x \neq 0$. Then

$$
B_{1} x=B_{1} E_{n} x=\sum_{k=1}^{\infty} \lambda_{k} E_{k} E_{n} x=\lambda_{n} E_{n} x=\lambda_{n} x .
$$

This shows that $\lambda_{n}$ is an eigenvalue of $B_{1}$. Since the spectrum of an operator is a closed set, it follows that $\sigma(A) \subset \sigma\left(B_{1}\right)$. This completes the proof of the lemma.

Proof of the first main theorem. We now come to the proof of this theorem, whose statement appears in $\S 1$. Let us define

$$
C_{1}=A-B_{1} \text {. }
$$

It follows readily from (3.2) and (1.1) that

$$
B^{k}=\sum_{n=1}^{\infty} \lambda_{n}^{k} E_{n} \quad \text { if } k \geqq 1 .
$$

From (3.1) and Lemma 2.2 we see that

$$
R_{\lambda}(A)=R_{\lambda}\left(B_{1}\right)+\sum_{n=2}^{\infty} \frac{Q_{n}}{\lambda^{n}},
$$

where

$$
Q_{n+1}=A^{n}-B_{1}^{n} \quad \text { if } n \geqq 1 .
$$

We observe that $Q_{2}=A-B_{1}=C_{1}$. We shall prove that

$$
Q_{2} B_{1}=B_{1} Q_{2}=0 \text {, }
$$

and that

$$
Q_{n+1}=Q_{2}^{n} \quad \text { if } n \geqq 2 \text {. }
$$

To prove (3.8) we start by observing that, since $\lambda_{n}$ is a first-order pole of $R_{\lambda}(A)$, we have the relation

$$
\left(A-\lambda_{n}\right) E_{n}=0,
$$

This is because $\left(A-\lambda_{n}\right) E_{n}$ is the coefficient of $\left(\lambda-\lambda_{n}\right)^{-2}$ in the Laurent expansion of $R_{\lambda}(A)$ about the point $\lambda=\lambda_{n}$; see formulas (5.8.1) and (5.8.6) in [3], p. 306. The same reasoning, or a direct argument from (3.2), shows that

$$
\left(B_{1}-\lambda_{n}\right) E_{n}=0
$$


It follows that

$$
Q_{2} E_{n}=\left(A-B_{1}\right) E_{n}=0,
$$

and from (3.2) we then deduce $Q_{2} B_{1}=0$. Since $E_{n}$ commutes with $A$ and $B_{1},(3.8)$ is now proved.

From (3.6) we see that

$$
I=(\lambda-A) R_{\lambda}\left(B_{1}\right)+(\lambda-A) \sum_{n=2}^{\infty} \frac{Q_{n}}{\lambda^{n}}
$$

when $\lambda \in \rho(A)$. Now $A=B_{1}+Q_{2}$, and hence

$$
(\lambda-A) R_{\lambda}\left(B_{1}\right)=\left(\lambda-B_{1}-Q_{2}\right) R_{\lambda}\left(B_{1}\right)=I-Q_{2} R_{\lambda}\left(B_{1}\right) .
$$

Going back to (3.12) and using the Neumann expansion for $R_{\lambda}\left(B_{1}\right)$, we find that, for all sufficiently large values of $\lambda$,

$$
0=-Q_{2} \sum_{n=0}^{\infty} \frac{B_{1}^{n}}{\lambda^{n+1}}+\left(\lambda-B_{1}-Q_{2}\right) \sum_{n=2}^{\infty} \frac{Q_{n}}{\lambda^{n}} .
$$

On comparing coefficients, we obtain the recurrence relations

$$
Q_{n+1}=\left(B_{1}+Q_{2}\right) Q_{n} \text { if } n \geqq 2 \text {. }
$$

In view of (3.8), the truth of (3.9) now follows at once by induction.

The series

$$
\sum_{n=2}^{\infty} \frac{Q_{n}}{\lambda^{n}}
$$

now takes the form

$$
\sum_{n=1}^{\infty} \frac{C_{1}^{n}}{\lambda^{n+1}}
$$

Since the series converges when $\lambda \neq 0$, it follows that $C_{1}$ is quasi-nilpotent, i.e. that

$$
\lim _{n \rightarrow \infty}\left\|C_{1}^{n}\right\|^{1 / n}=0
$$

and that $\sigma\left(C_{1}\right)$ is the single point 0 . Moreover, in view of the form of the Neumann expansion, the series in (3.14) has the value

$$
R_{\lambda}\left(C_{1}\right)-\frac{I}{\lambda} .
$$

In connection with these arguments, see $\S 5.2$ of [3]. The proof of the first main theorem is now completed. 
4. The case $v_{n}=p$ for all $n$. According to Lemma 2.2, the form of $R_{\lambda}(A)$ in this case is

$$
\begin{aligned}
R_{\lambda}(A)= & \frac{I}{\lambda}+\frac{A}{\lambda^{2}}+\cdots+\frac{A^{p-1}}{\lambda^{p}} \\
& +\sum_{n=1}^{\infty}\left[\frac{1}{\lambda-\lambda_{n}}-\frac{1}{\lambda}-\cdots-\frac{\lambda_{n}^{p-1}}{\lambda^{p}}\right] E_{n} \\
& +\sum_{n=p}^{\infty} \frac{1}{\lambda^{n+1}}\left[A^{n}-\sum_{k=1}^{\infty} \lambda_{k}^{n} E_{k}\right] .
\end{aligned}
$$

We remark in passing that if we merely assume $v_{n} \leqq p$ for all $n$, $R_{\lambda}(A)$ can still be brought to the form (4.1), so that one might as well assume $v_{n}=p$ for all $n$. Also, the expansion (4.1) will be valid if lim $\sup _{n \rightarrow \infty} \nu_{n}=p$, for in that case $v_{n} \leqq p$ when $n$ is sufficiently large, and it is possible, by a finite number of rearrangements, to arrange matters just as they would be if we had $v_{n} \leqq p$ for all values of $n$.

Now we define an operator $B_{p}$ by the formula

$$
B_{p}=\sum_{n=1}^{\infty} \lambda_{n}^{p} E_{n},
$$

and we proceed to prove the second main theorem, as stated in $\S 1$.

By the spectral mapping theorem (see $\S 5.71$ of [3]) we know that $\sigma\left(A^{p}\right)$ consists of 0 and the points $\lambda_{1}^{p}, \lambda_{2}^{p}, \lambda_{3}^{p}, \cdots$. We shall compute the resolvent of $A^{p}$. If $\lambda$ is different from 0 and all of the $\lambda_{n}^{p}$, we know by the operational calculus that

$$
\left(\lambda-A^{p}\right)^{-1}=f(A), \text { where } f(t)=\left(\lambda-t^{p}\right)^{-1} ;
$$

see $\S 5-6$ of [3], especially Theorem 5.6-B. Thus

$$
\left(\lambda-A^{p}\right)^{-1}=\frac{1}{2 \pi i} \oint \frac{1}{\lambda-t^{p}} R_{t}(A) d t,
$$

where the integral is extended over the boundary of a Cauchy domain which contains $\sigma(A)$ and whose closure excludes all the $p$ th roots of $\lambda$. When we use (4.1) to give $R_{t}(A)$, and compute the integral in (4.3) by term-by-term integration, the result is

$$
\begin{aligned}
\left(\lambda-A^{p}\right)^{-1}=\frac{I}{\lambda} & +\sum_{n=1}^{\infty}\left[\frac{1}{\lambda-\lambda_{n}^{p}}-\frac{1}{\lambda}\right] E_{n} \\
& +\sum_{r=1}^{\infty} \frac{1}{\lambda^{r+1}}\left[A^{r p}-\sum_{k=1}^{\infty} \lambda_{k}^{r p} E_{k}\right] .
\end{aligned}
$$

It is a simple matter to show that the series with index $n$ converges 
uniformly on compact subsets of $\rho\left(A^{p}\right)$, while the series with index $r$ converges whenever $\lambda \neq 0$.

A comparison of (4.4) and (3.1) now shows that the first main theorem is applicable to $A^{p}$. We have $A^{p}, \lambda_{n}^{p}, B_{p}$ in place of $A, \lambda_{n}, B_{1}$, respectively. There is one minor point which calls for comment. The points $\lambda_{1}^{p}, \lambda_{2}^{p}, \cdots$ need not all be distinct, even though the points $\lambda_{1}, \lambda_{2}, \cdots$ are all distinct. This is not an essential matter, however. If several of the $\lambda_{n}^{p}$ are the same, the terms which involve them can be combined, and the sum of the corresponding $E_{n}$ 's is a projection. This concludes the proof of the second main theorem.

5. Operations of finite type $p$. Let us start out by assuming that $A$ is such that $v_{n}=p$ for all values of $n$, so that (4.1) holds. Let us also assume that

$$
A^{p}=\sum_{k=1}^{\infty} \lambda_{k}^{p} E,
$$

so that the $C_{p}$ of (1.13) is 0 in this case. By (3.10) we know that $A E_{k}=\lambda_{k} E_{k}$. Hence from (5.1) it follows by induction that

$$
A^{n}=\sum_{k=1}^{\infty} \lambda_{k}^{n} E_{k}
$$

if $n \geqq p$. We then see from (4.1) that $R_{\lambda}(A)$ has the form

$$
R_{\lambda}(A)=\frac{I}{\lambda}+\cdots+\frac{A^{p-1}}{\lambda^{p}}+\sum_{n=1}^{\infty}\left[\frac{1}{\lambda-\lambda_{n}}-\frac{1}{\lambda}-\cdots-\frac{\lambda_{n}^{p-1}}{\lambda^{p}}\right] E_{n},
$$

which means that $A$ is of finite type $\leqq p$ (see $\S 1$ ). Conversely, from (5.2), written more conveniently in the form (1.16), we readily deduce (5.1) (multiply by $\lambda^{p}$ and integrate around a contour enclosing $\sigma(A)$ ).

Let us now undertake the proof of the third main theorem, as stated in $\S 1$. The motivation for this theorem is an expansion theorem for meromorphic functions, due originally to Cauchy, but conveniently accessible in Titchmarsh's text [4] (§3.2 and $\S 3.21)$.

Let $\lambda$ be confined to a compact set $S$ lying in $\rho(A)$. Let $\Gamma$ be a circle with center at the origin, large enough to enclose $S, \sigma(A)$, and all the contours $C_{1}, C_{2}, \cdots$. Consider the integrals (in the counterclockwise sense)

$$
\begin{gathered}
I_{n}=\frac{1}{2 \pi i} \int_{\sigma_{n}} \frac{t^{p}}{\lambda-t} R_{t}(A) d t, \\
J=\frac{1}{2 \pi i} \int_{\Gamma} \frac{t^{p}}{\lambda-t} R_{t}(A) d t,
\end{gathered}
$$


Evidently $J-I_{n}$ is equal to the sum of the residues of the integrand at the points $\lambda, \lambda_{1}, \cdots, \lambda_{n}$. This sum of residues is

$$
-\lambda^{p} R_{\lambda}(A)+\sum_{k=1}^{n} \frac{\lambda_{k}^{p}}{\lambda-\lambda_{k}} E_{k}
$$

We can calculate the value of $J$, for on $\Gamma$

$$
R_{t}(A)=\frac{I}{t}+\frac{A}{t^{2}}+\frac{A^{2}}{t^{3}}+\cdots
$$

and

$$
\frac{t^{p}}{\lambda-t}=-t^{p-1}\left(1+\frac{\lambda}{t}+\frac{\lambda^{2}}{t^{2}}+\cdots\right) \text {. }
$$

It then follows readily that

$$
J=-\left(A^{p-1}+\lambda A^{p-2}+\cdots+\lambda^{p-1} I\right) .
$$

Hence

$$
R_{\lambda}(A)=\frac{I}{\lambda}+\frac{A}{\lambda^{2}}+\cdots+\frac{A^{p-1}}{\lambda^{p}}+\sum_{k=1}^{n} \frac{\lambda_{k}^{p}}{\lambda^{p}\left(\lambda-\lambda_{k}\right)} E_{k}+\frac{1}{\lambda^{p}} I_{n} .
$$

We shall now prove that $I_{n} \rightarrow 0$ uniformly with respect to $\lambda$ in $S$. This will complete the proof of the third main theorem. Using the notation established in the theorem itself, we see that

$$
\left|I_{n}\right| \leqq \frac{\varepsilon_{n}^{p}}{2 \pi\left(|\lambda|-\varepsilon_{n}\right)} l\left(C_{n}\right) M_{n},
$$

provided that $\varepsilon_{n}<|\lambda|$. Since $l\left(C_{n}\right)=O\left(\varepsilon_{n}\right), \varepsilon_{n} \rightarrow 0$, and $S$ is a compact set not containing the origin, the result now follows from the assumption that $\varepsilon_{n}^{p+1} M_{n} \rightarrow 0$.

6. Some sufficient conditions for operators of finite type 1. Let us suppose that $E_{1}, E_{2}, \cdots$ are bounded projections on $X$ such that $E_{n} \neq 0$ and $E_{m} E_{n}=0$ if $m \neq n$. Then $E_{n} \neq I$. For, $E_{n}=I$ for some $n$ would imply $0=E_{n} E_{n+1}=E_{n+1}$. Next, let us suppose $\left\{\lambda_{n}\right\}$ is a sequence of distinct constants such that the series

$$
\sum_{n=1}^{\infty} \lambda_{n} E_{n}
$$

is convergent in $[X]$, (This implies that $\lambda_{n} \rightarrow 0$.) Let

$$
B=\sum_{n=1}^{\infty} \lambda_{n} E_{n},
$$


We ask the question: What further conditions, if any, are required to assure us that $B$ is of finite type 1 ? The necessary and sufficient condition for this is that all points except 0 and the $\lambda_{n}$ 's be in $\rho(B)$, and that

$$
R_{\lambda}(B)=\frac{I}{\lambda}+\sum_{n=1}^{\infty}\left[\frac{1}{\lambda-\lambda_{n}}-\frac{1}{\lambda}\right] E_{n} .
$$

The proof of Lemma 3.1, if we re-read it in the present context, allows us to assert the following:

LEMma 6.1. With the assumptions made in the first paragraph of this section, the operator $B$ defined by (6.1) is of finite type 1 if and only if the series on the right in (6.2) converges uniformly on compact subsets of the $\lambda$-plane which do not contain 0 or any of the $\lambda_{n}$ 's.

We shall give two types of conditions which enable us to utilize the foregoing lemma.

TheOREM 6.2. In addition to the assumptions made in the paragraph leading up to (6.1), let us assume that the series

$$
\sum_{n=1}^{\infty}\left|\lambda_{n+1}-\lambda_{n}\right|
$$

is convergent. Then the operator $B$ defined by (6.1) is of finite type 1 .

Proof. The argument is like that in certain classical tests for nonabsolute convergence (see, e.g. [1], pp. 25-26 and pp. 98-100). Let $S$ be a compact subset of the plane of the type mentioned in Lemma 6.1. Then there is a positive constant $M$ such that $\left|\left(\lambda-\lambda_{n}\right)^{-1}\right| \leqq M$ for all $n$ if $\lambda$ is in $S$. Let

$$
\begin{aligned}
& r_{0}=\sum_{n=1}^{\infty} \lambda_{n} E_{n}, \\
& r_{k}=\sum_{n=k+1}^{\infty} \lambda_{n} E_{n} . \quad k=1.2, \cdots
\end{aligned}
$$

Let

$$
v_{n}(\lambda)=\frac{1}{\lambda-\lambda_{n}}
$$

Then $\left|v_{n}(\lambda)\right| \leqq M$ when $\lambda$ is in $S$, and it is easy to see that

$$
\sum_{n=1}^{\infty}\left|v_{n+1}(\lambda)-v_{n}(\lambda)\right| \leqq M^{2} \sum_{n=1}^{\infty}\left|\lambda_{n+1}-\lambda_{n}\right| \text {. }
$$

It suffices to prove that the series 


$$
\sum_{n=1}^{\infty} \lambda_{n} v_{n}(\lambda) E_{n}
$$

converges uniformly on $S$. Now, if $p \geqq 1$,

$$
\begin{aligned}
\sum_{k=1}^{p} \lambda_{n+k} v_{n+k}(\lambda) E_{n+k}= & \sum_{k=1}^{p}\left\{v_{n+k}(\lambda)-v_{n+k-1}(\lambda)\right\} r_{n+k-1} \\
& +r_{n} v_{n}(\lambda)-r_{n+p} v_{n+p}(\lambda)
\end{aligned}
$$

and so

$$
\begin{aligned}
\left\|\sum_{k=1}^{n} \lambda_{n+k} v_{n+k}(\lambda) E_{n+k}\right\| & \leqq \sup _{k \geq n}\left\|r_{k}\right\|\left\{\sum_{n=1}^{\infty}\left|v_{n+1}(\lambda)-v_{n}(\lambda)\right|+2 M\right\} \\
& \leqq \sup _{k \geqq n}\left\|r_{k}\right\|\left\{M^{2} \sum_{n=1}^{\infty}\left|\lambda_{n+1}-\lambda_{n}\right|+2 M\right\} .
\end{aligned}
$$

Since $r_{n} \rightarrow 0$ as $n \rightarrow \infty$, this finishes the proof.

Observe that the geometrical meaning of the convergence of the series (6.3) is that the polygonal path formed by joining $\lambda_{1}, \lambda_{2}, \lambda_{3}, \cdots$ in succession shall have finite length. This is true, in particular, if the $\lambda_{n}$ 's approach the origin monotonely along some ray.

THEOREM 6.3. Suppose that $\left\{E_{n}\right\}$ is a sequence of projections on the Banach space $X$, of such a character that $E_{n} \neq 0$ and $E_{n} E_{m}=0$ if $m \neq n$. Suppose further that there is some constant $M$ such that, for every finite set of constants $c_{1}, \cdots, c_{n}$, we have

$$
\left\|\sum_{k=1}^{n} c_{k} E_{k}\right\| \leqq \sup _{i}\left|c_{i}\right| \text {. }
$$

Then, if $\left\{\lambda_{n}\right\}$ is any sequence of distinct constants such that $\lambda_{n} \rightarrow 0$, the series

$$
B=\sum_{n=1}^{\infty} \lambda_{n} E_{n}
$$

converges in $[X]$ and defines an operator of finite type 1.

Proof. The convergence of (6.5) results from (6.4), for, it $m<n$

$$
\left\|\sum_{k=m+1}^{n} \lambda_{k} E_{k}\right\| \leqq M \sup _{m<i \leqq n}\left|\lambda_{i}\right|
$$

and the Cauchy convergence condition is satisfied, since $\lambda_{n} \rightarrow 0$.

Now let $S$ be any compact set which does not contain 0 or any of the $\lambda_{n}$ 's. Then

$$
\inf \left|\lambda-\lambda_{n}\right|=\delta>0 \quad(\lambda \in S, n=1,2, \cdots) .
$$

Hence, for $\lambda \in S$ and $m<n$ 


$$
\left\|\sum_{k=m+1}^{n} \frac{\lambda_{k}}{\lambda-\lambda_{k}} E_{k}\left|\| \leqq \frac{M}{\delta} \sup _{m<k \leqq n}\right| \lambda_{k} \mid .\right.
$$

The uniform convergence on $S$ of

$$
\sum_{n=1}^{\infty} \frac{\lambda_{n}}{\lambda-\lambda_{n}} E_{n}
$$

is thus established, and the conclusion follows from Lemma 6.1.

The condition (6.4) is automatically satisfied (with $M=1$ ) if $\left\{E_{n}\right\}$ is a sequence of nonzero mutually orthogonal projections in Hilbert space. For, in this case we can first of all establish that

$$
\sum_{n=1}^{\infty}\left\|E_{n} x\right\|^{2} \leqq\|x\|^{2}
$$

for each $x$. In fact, let

$$
v_{k}=\frac{E_{k} x}{\left\|E_{k} x\right\|} \text { if } E_{k} x \neq 0, \quad v_{k}=0 \quad \text { otherwise } .
$$

The $\left(v_{j}, v_{k}\right)=0$ if $j \neq k$, and $\left\|v_{k}\right\|$ is either 0 or 1 . Hence, since an easy calculation shows that $\left(x, v_{k}\right)=\left\|E_{k} x\right\|$, we have

$$
\sum_{n=1}^{\infty}\left\|E_{n} x\right\|^{2}=\sum_{n=1}^{\infty}\left|\left(x, v_{n}\right)\right|^{2} \leqq\|x\|^{2},
$$

by the Bessel inequality. It now follows, using (6.6), that

$$
\begin{aligned}
\left\|\sum_{k=1}^{n} c_{k} E_{k} x\right\|^{2} & =\sum_{k=1}^{n}\left|c_{k}\right|^{2}\left\|E_{k} x\right\|^{2} \\
& \leqq\left(\sum_{k=1}^{n}\left\|E_{k} x\right\|^{2}\right) \sup _{k}\left|c_{k}\right|^{2} \\
& \leqq\|x\|^{2} \sup _{k}\left|c_{k}\right|^{2} .
\end{aligned}
$$

From this we infer

$$
\left\|\sum_{k=1}^{n} c_{k} E_{k}\right\| \leqq \sup _{k}\left|c_{k}\right|
$$

7. Some open questions. Let us consider an operator $B$ as defined by (6.1), and let us assume nothing more than is specified in the first paragraph of $\S 6$. It is not clear that this is enough to give us an operator of finite type 1 . Indeed, the nature of $\sigma(B)$ is not clear. We can prove that each $\lambda_{n}$ is an eigenvalue, but it is not evident that a $\lambda$ different from 0 and all the $\lambda_{n}$ 's is in $\rho(B)$. We shall prove, however, that for such a the range of $\lambda-B$ is dense in $X$ and consists of exactly those elements $y \in X$ for which the series 


$$
\sum_{n=1}^{\infty} \frac{\lambda_{n}}{\lambda-\lambda_{n}} E_{n} y
$$

is convergent. Moreover, such a $\lambda$ is not an eigenvalue of $B$.

Proof of the italicized assertions: With $\lambda$ as indicated, suppose $y$ is in the range of $\lambda-B$, so that $(\lambda-B) x=y$ for some $x$. Then

$$
\begin{aligned}
\lambda x-\sum_{n=1}^{\infty} \lambda_{n} E_{n} x & =y, \\
\lambda E_{j} x-\lambda_{j} E_{j} x & =E_{j} y, \\
E_{j} x & =\frac{E_{i} y}{\lambda-\lambda_{j}} .
\end{aligned}
$$

Therefore

$$
\lambda x-\sum_{n=1}^{\infty} \frac{\lambda_{n} E_{n} y}{\lambda-\lambda_{n}}=y,
$$

or

$$
x=\frac{1}{\lambda} y+\sum_{n=1}^{\infty} \frac{\lambda_{n}}{\lambda\left(\lambda-\lambda_{n}\right)} E_{n} y
$$

Thus the series in (7.2) converges, and (7.2) defines $x$ as the unique vector such that $(\lambda-B) x=y$. This guarantees that $\lambda$ is not an eigenvalue of $B$.

Suppose now that $y$ is a vector such that the series (7.1) is convergent ( $\lambda$ being fixed, different from 0 and all the $\lambda_{n}$ 's). Define a vector $x$ by the series (7.2). A direct calculation shows that

$$
E_{j} x=\frac{E_{j} y}{\lambda-\lambda_{j}}
$$

whence

$$
x=\frac{1}{\lambda} y+\sum_{n=1}^{\infty} \frac{\lambda_{n}}{\lambda} E_{n} x,
$$

or $(\lambda-B) x=y$.

We have now proved all of the italicized assertions except the assertion that the range of $\lambda-B$ is dense in $X$. To do this we consider the first and second conjugate spaces $X^{\prime}, X^{\prime \prime}$, and the conjugate operators

$$
B^{\prime}=\sum_{n=1}^{\infty} \lambda_{n} E_{n}^{\prime}, \quad B^{\prime \prime}=\sum_{n=1}^{\infty} \lambda_{n} E_{n}^{\prime \prime}
$$


The original assumptions about $B$ and the $E_{n}$ 's carry over to these conjugate operators, and, accordingly, a $\lambda$ different from 0 and all the $\lambda_{n}$ 's is not an eigenvalue of $B^{\prime}$ or of $B^{\prime \prime}$. Now, if the range of $\lambda-B$ were not dense in $X$, this would imply that $\lambda$ is an eigenvalue of $B^{\prime}$ (see Theorem 4.6-E, p. 226, or the state diagram, p. 237, in [3]). Hence the range of $\lambda-B$ is dense in $X$, and likewise the range of $\lambda-B^{\prime}$ is dense in $X^{\prime}$. A perusal of the state diagram in [3] now shows that a $\lambda$ different from 0 and all the $\lambda_{n}$ 's, if indeed such a $\lambda$ can be in $\sigma(B)$, is in the continuous spectrum of $B$. That is, $\lambda-B$ has range dense in $X$, but the inverse is discontinuous. Likewise for $\lambda$ in relation to $B^{\prime}$.

Supplementary note: After this paper had been accepted for publication, a discussion of its contents with Mr. Earl Berkson led him to settle the problems of this final section very neatly. His results are in the immediately following paper. Mr. Berkson also spotted some ambiguity in the concept of an operator of finite type. His comments of clarification, and his interesting example of a resolvent with a MittagLeffler development which is not unconditionally convergent, should be noted by readers of my paper.

\section{REFERENCES}

1. E. T., Copson, An introduction to the theory of functions of a complex variable, Oxford, 1935.

2. N. Dunford, Spectral operators, Pacific J. Math., 4 (1954), 321-354.

3. Angus E. Taylor, Introduction to functional analysis, John Wiley, New York, 1958.

4. E. C. Titchmarsh, Theory of functions, Oxford, 1932.

THE UNIVERSITY OF CALIFORNIA

LOS ANGELES 


\section{PACIFIC JOURNAL OF MATHEMATICS}

\section{EDITORS}

\author{
David Gilbarg \\ Stanford University \\ Stanford, California \\ F. H. Brownell \\ University of Washington \\ Seattle 5 , Washington
}

\author{
A. L. Whiteman \\ University of Southern California \\ Los Angeles 7, California \\ L. J. PAIGE \\ University of California \\ Los Angeles 24, California
}

\section{ASSOCIATE EDITORS}

\author{
E. F. BECKENBACH \\ T. M. CHERRY \\ D. DERRY
}
E. HEWITT
A. HORN
L. NACHBIN
M. OHTSUKA
H. L. ROYDEN
M. M. SCHIFFER

E. SPANIER

E. G. STRAUS

F. WOLF

\section{SUPPORTING INSTITUTIONS}

\author{
UNIVERSITY OF BRITISH COLUMBIA \\ CALIFORNIA INSTITUTE OF TECHNOLOGY \\ UNIVERSITY OF CALIFORNIA \\ MONTANA STATE UNIVERSITY \\ UNIVERSITY OF NEVADA \\ NEW MEXICO STATE UNIVERSITY \\ OREGON STATE COLLEGE \\ UNIVERSITY OF OREGON \\ OSAKA UNIVERSITY \\ UNIVERSITY OF SOUTHERN CALIFORNIA
}

\author{
STANFORD UNIVERSITY \\ UNIVERSITY OF TOKYO \\ UNIVERSITY OF UTAH \\ WASHINGTON STATE COLLEGE \\ UNIVERSITY OF WASHINGTON

AMERICAN MATHEMATICAL SOCIETY
CALIFORNIA RESEARCH CORPORATION
HUGHES AIRCRAFT COMPANY
SPACE TECHNOLOGY LABORATORIES
NAVAL ORDNANCE TEST STATION

Mathematical papers intended for publication in the Pacific Journal of Mathematics should be typewritten (double spaced), and the author should keep a complete copy. Manuscripts may be sent to any one of the four editors. All other communications to the editors should be addressed to the managing editor, L. J. Paige at the University of California, Los Angeles 24, California. ..

50 reprints per author of each article are furnished free of charge; additional copies may be obtained at cost in multiples of 50 .

The Pacific Journal of Mathematics is published quarterly, in March, June, September, and December. The price per volume (4 numbers) is $\$ 12.00$; single issues, $\$ 3.50$. Back numbers are available. Special price to individual faculty members of supporting institutions and to individual members of the American Mathematical Society: $\$ 4.00$ per volume; single issues, $\$ 1.25$.

Subscriptions, orders for back numbers, and changes of address should be sent to Pacific Journal of Mathematics, 2120 Oxford Street, Berkeley 4, California.

Printed at Kokusai Bunken Insatsusha (International Academic Printing Co., Ltd.), No. $6_{4}$ 2-chome, Fujimi-cho, Chiyoda-ku, Tokyo, Japan.

PUBLISHED BY PACIFIC JOURNAL OF MATHEMATICS, A NON-PROFIT CORPORATION

The Supporting Institutions listed above contribute to the cost of publication of this Journăl, but they are not owners or publishers and have no responsibility for its content or policies. 


\section{Pacific Journal of Mathematics}

\section{Vol. 10, No. $3 \quad$ November, 1960}

Glen Earl Baxter, An analytic problem whose solution follows from a simple

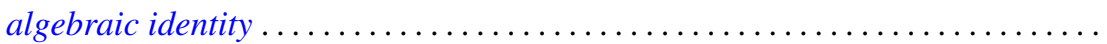

Leonard D. Berkovitz and Melvin Dresher, A multimove infinite game with linear payoff. .

Earl Robert Berkson, Sequel to a paper of A. E. Taylor ......................

Gerald Berman and Robert Jerome Silverman, Embedding of algebraic systems.... 767

Peter Crawley, Lattices whose congruences form a boolean algebra . . . . . ...... 777

Robert E. Edwards, Integral bases in inductive limit spaces . . . . . . . . . . . . . . .

Daniel T. Finkbeiner, II, Irreducible congruence relations on lattices . . . . . . . . . .

William James Firey, Isoperimetric ratios of Reuleaux polygons . . . . . . . . . . . 787

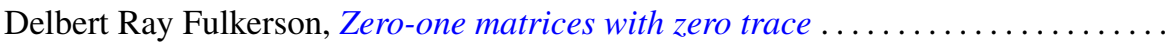

Leon W. Green, A sphere characterization related to Blaschke's conjecture........

Israel (Yitzchak) Nathan Herstein and Erwin Kleinfeld, Lie mappings in

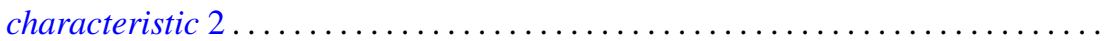

Charles Ray Hobby, A characteristic subgroup of a $p$-group .................

R. K. Juberg, On the Dirichlet problem for certain higher order parabolic

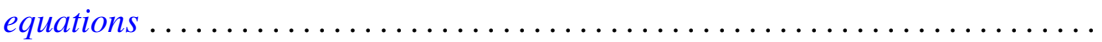

Melvin Katz, Infinitely repeatable games ......................

Emma Lehmer, On Jacobi functions . . . . . . . . . . . . . . . . . . . . . . . . .

D. H. Lehmer, Power character matrices

Henry B. Mann, A refinement of the fundamental theorem on the density of the sum of two sets of integers.

Marvin David Marcus and Roy Westwick, Linear maps on skew symmetric matrices: the invariance of elementary symmetric functions . .

Richard Dean Mayer and Richard Scott Pierce, Boolean algebras with ordered

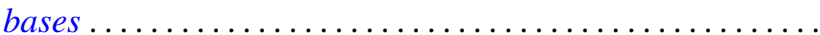

Trevor James McMinn, On the line segments of a convex surface in $E_{3} \ldots$

Frank Albert Raymond, The end point compactification of manifolds ..

Edgar Reich and S. E. Warschawski, On canonical conformal maps of regions of arbitrary connectivity

Marvin Rosenblum, The absolute continuity of Toeplitz's matrices...

Lee Albert Rubel, Maximal means and Tauberian theorems . .

Helmut Heinrich Schaefer, Some spectral properties of positive linear operators

Jeremiah Milton Stark, Minimum problems in the theory of pseudo-conformal transformations and their application to estimation of the curvature of the invariant metric.

Robert Steinberg, The simplicity of certain groups ...

Hisahiro Tamano, On paracompactness. .

Angus E. Taylor, Mittag-Leffler expansions and spectral theory .

Marion Franklin Tinsley, Permanents of cyclic matrices ...... . 